\title{
Message from the EiC
}

Machine intelligence is a fast-developing field and often used as a synonym for artificial intelligence but with a stronger application orientation. Machine intelligence algorithms are not forms of alchemy, but rather useful tools of improving the world we live in. With easier access to data and more affordable computational power, discoveries in research labs are being transformed into real-world applications and therefore creating enormous social and economic values. From medical imaging to autonomous driving, we have been witnesses to the development of machine intelligence unfolding at such a stunning speed. Making scientific breakthroughs has always been a bumpy journey and many underpinning challenges are yet to be overcome. We have come to a point where pushing the developing boundaries of machine intelligence requires contributions of diverse areas such as Brain and Cognitive Sciences, Psychology, Mechanics, Network Technology, Big Data and Cloud Computing. We feel urged to provide a dedicated platform that can bring and share the perspectives of great minds, thus making a big difference for bettering human life.

The expansion of machine intelligence research scope has led to an unprecedented increase of exciting innovations and findings. This change can also be tracked in the predecessor of Machine Intelligence Research (MIR), namely International Journal of Automation and Computing (IJAC), in which nearly 90 percent of published articles pertain to machine intelligence. Besides the academic community, the impact of machine intelligence also goes to the industrial world and indeed the whole society by instilling greater productive forces and bringing a new round of industrial revolution.

Under such circumstances, to better reflect the focus of IJAC submissions and to better satisfy the needs of the human society, we have taken a leap of faith and decided to rename the International Journal of Automation and Computing as Machine Intelligence Research from January 2022 after consulting with members of the IJAC community. We want to be at the forefront of publishing machine intelligence research.

As a new journal with a successful predecessor, Machine Intelligence Research aims to publish high-quality papers on original theoretical and experimental research,

\footnotetext{
Editorial

Colored figures are available in the online version at https://link. springer.com/journal/11633

(C) Institute of Automation, Chinese Academy of Sciences and Springer-Verlag GmbH Germany, part of Springer Nature 2022
}

targets special issues on emerging topics, and strives to bridge the gap between theoretical research and practical applications. Topics of interest include but are not limited to:

- AI Fundamentals

- Brain-inspired Intelligence

- Pattern Recognition \& Machine Learning

- Machine Vision

- Speech and Language Processing

- Robotics

- Knowledge Management \& Data Mining

- Applications of Machine Intelligence

We welcome reviews, research articles, perspectives and correspondences exploring the above topics.

We strive for fair peer review, fast publication and wide publicity. We have gathered a strong group of experts to form a highly motivated Editorial Board with Associate Editors-in-Chief, International Advisors, Associate Editors and myself as the Editor-in-Chief. The Editorial Board will handle submissions in a timely fashion and will spare no efforts in strengthening MIR's global authorship and promoting MIR to significantly increase the number of worldwide readers. We are privileged to have Professors Yike Guo (Hong Kong Baptist University, China and Imperial College London, UK), Brian C. Lovell (The University of Queensland, Australia) and Liang Wang (Chinese Academy of Sciences, China) as Associate Editors-in-Chief, and Professors Rama Chellappa (University of Maryland, USA), Toshio Fukuda (Nagoya University, Japan), Anil K. Jain (Michigan State University, USA), Tomaso Poggio (Massachusetts Institute of Technology, USA), Mu-ming Poo (Chinese Academy of Sciences, China), Bo Xu (Chinese Academy of Sciences, China) and Bo Zhang (Tsinghua University, China) to serve on the Advisory Board.

I would like to express my deep gratitude to all members of the Editorial Board. They are highly qualified and experienced. They will preview the manuscripts in their areas of expertise, make initial decisions about the suitability for further peer review, allocate reviewers, and then give suggestions on whether a paper is suitable for publication. We hope that this process will ensure that each manuscript receives excellent reviews from professionals, and that decisions about the suitability for publication are agreed upon among MIR reviewers, Associate Editors, Associate Editors-in-Chief and Editor-in-Chief. Together with our readership, the whole MIR team will strive to fulfill the mission of disseminating highest-quality origin- 
al research and information and to make the journal a highest-quality forum for sharing research experiences and reflections.

MIR's sponsor, the Institute of Automation of the Chinese Academy of Sciences (CASIA) is always our strong backing. As a well-established national institute in the field of machine intelligence, CASIA focuses on braininspired intelligence and autonomous intelligent systems. By cooperating with various international research institutions, CASIA has established joint laboratories and engineering centers, contributing significantly to the academic communications of the international AI community. With strong support from CASIA, Machine Intelligence Research will always be rooted on a solid foundation and best positioned to bring in new ideas, open wider scope and facilitate international cooperation.

We have embarked on this brand-new journey with Machine Intelligence Research. We will work diligently together to advance MIR to a high level of excellence. We hope our readers will find the content of the journal timely, engaging, and thought-provoking, and we look forward to your contributions.

Tieniu Tan

Editor-in-Chief of Machine Intelligence Research

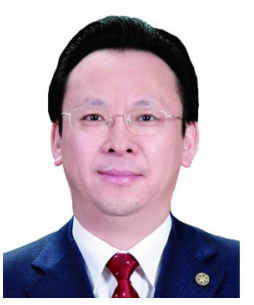

Tieniu Tan received his B.Sc. degree in electronic engineering from Xi'an Jiaotong University, China, in 1984, and his M.Sc. and Ph.D. degrees in electronic engineering from Imperial College London, UK, in 1986 and 1989, respectively. In October 1989, he joined the Computational Vision Group at the Department of Computer Science, The University of Reading, Reading, UK, where he worked as a Research Fellow, Senior Research Fellow and Lecturer. In January 1998, he returned to China to join the National Laboratory of Pattern Recognition (NLPR), Institute of Automation of the Chinese Academy of Sciences (CAS), Beijing, China, where he is currently a Professor and the Director of Center for Research on Intelligent Perception and Computing (CRIPAC), and was former Director (1998-2013) of the NLPR, Director General of the Institute (2000-2007), and Deputy Secretary-General (2007-2015) and Vice President (2015-2016) of CAS. He has published 15 edited books or monographs and more than 600 research papers in refereed international journals and conferences in the areas of image processing, computer vision and pattern recognition. Dr. Tan is a Fellow of CAS, TWAS (The World Academy of Sciences for the advancement of science in developing countries), IEEE and IAPR (the International Association of Pattern Recognition), an International Fellow of the UK Royal Academy of Engineering and a Corresponding Member of the Brazilian Academy of Sciences.

E-mail: tnt@nlpr.ia.ac.cn 